\title{
La violencia y la salud pública
}

\author{
Alberto Pellegrini Filho ${ }^{1}$
}

La Organización Panamericana de la Salud (OPS), a lo largo de sus 97 años de existencia, viene cooperando con los países de las Américas a fin de producir y utilizar los conocimientos necesarios para promover la salud y solucionar los problemas sanitarios de mayor relevancia. Siguiendo esta tradición, el Programa de Subvenciones para la Investigación en Salud de la OPS promueve y apoya estudios de carácter multicéntrico. Por medio de estos estudios, grupos de investigación en diversos países de la Región desarrollan conjuntamente un proyecto de investigación sobre un problema prioritario de ámbito regional, o sea, un problema común a varios países cuyo origen e impacto transciende las fronteras nacionales y cuya solución precisa un esfuerzo colaborativo.

Los grupos de investigación que trabajan en estos proyectos multicéntricos elaboran en conjunto un protocolo común y, a lo largo de todo el proceso de investigación, establecen lazos de intercambio y cooperación. Esta modalidad de trabajo no solo permite realizar análisis comparativos de mayor poder explicativo para fundamentar el diseño de las intervenciones, sino que también contribuye a fortalecer la capacidad para realizar investigaciones mediante la creación de redes de colaboración.

En 1996, la Coordinación de Investigaciones de la División de Salud y Desarrollo Humano, junto con la División de Promoción y Protección de la Salud de la OPS, empezaron a desarrollar el proyecto multicéntrico sobre actitudes y normas culturales relacionadas con la violencia a partir de un protocolo inicial elaborado por Alfred McAllister del Centro Colaborador de la OPS en Promoción de la Salud de la Universidad de Texas en Houston, Estados Unidos de América. Tras una serie de reuniones de trabajo y de pruebas piloto de instrumentos, la investigación se llevó a cabo en ocho ciudades: San Salvador, El Salvador; San José, Costa Rica; Caracas, Venezuela; Cali, Colombia; Río de Janeiro y Salvador, Brasil; Santiago, Chile, y Madrid, España. Los gastos para recabar datos fueron en buena

Coordinación de Investigaciones, Organización Panamericana de la Salud, Washington, D.C., Estados Unidos de América. parte financiados por otras fuentes locales e internacionales, lo que revela la capacidad de este tipo de proyectos para movilizar recursos.

En este número especial de la Revista Panamericana de Salud Pública/Pan American Journal of Public Health tenemos la satisfacción de presentar una serie de análisis y estudios comparativos realizados con los datos obtenidos en las diversas ciudades, así como los informes específicos correspondientes a algunas de ellas. En números posteriores de la Revista/Journal se publicarán otros informes que forman parte de este estudio multicéntrico.

A pesar de las deficiencias de los sistemas de información sobre la violencia en los países de la Región, estudios recientes sobre la magnitud del problema - expresada en defunciones y costos- revelan su enorme importancia y la gran heterogeneidad de las situaciones originadas por la violencia. De las 116623 muertes causadas por homicidios en la Región en 1994 (29 países), 88,6\% se notificaron en hombres y $11,4 \%$, en mujeres. Las tasas de mortalidad por homicidio en ese mismo año variaron de 6,2 por 100000 en el Cono Sur a 51,9 por 100000 en la Región Andina. Cabe señalar que esta última cifra fue dos veces mayor que la notificada en 1984. En Colombia, el homicidio fue la primera causa de muerte en 1994 en los hombres y representó 41\% de todos los años potenciales de vida perdidos (APVP) (1). Se estima, además, que cerca de $10 \%$ del producto interno bruto de la Región se destina a sufragar gastos derivados de la violencia (2).

Aunque la violencia ha sido objeto de numerosos estudios desde el punto de vista de diversas disciplinas, su abordaje como problema de salud pública es relativamente reciente. La violencia puede y debe ser tratada como un problema de salud pública, no solo porque produce directamente lesiones y defunciones, sino por su influencia en el deterioro del entramado de relaciones sociales de solidaridad y cooperación que hoy se suele denominar "capital social". Varios estudios recientes demuestran que no son necesariamente las sociedades más ricas las que tienen mejores niveles de salud, sino las que son más igualitarias, es decir, las que también poseen alta cohesión social y una fuerte vida comunitaria (3). Las investigaciones indican cómo las desigual- 
dades y la violencia afectan a este tejido social tan importante para la salud colectiva.

La violencia nos interesa como problema de salud pública por su efecto deletéreo en las condiciones generales de salud y bienestar de las poblaciones. Empero, sería inútil tener la voluntad de enfrentar un problema de tal complejidad si no dispusiéramos de las nuevas herramientas que han brindado los avances de la teoría y la práctica de la salud pública. Es muy importante reparar en la diferencia entre tratar la violencia como un problema de salud pública y aplicar el enfoque de la salud pública al problema de la violencia. Los que proponen la última opción suelen identificar al enfoque de la salud pública con el enfoque de riesgo, según el cual los factores determinantes de carácter sociocultural suelen analizarse a partir de unas pocas variables tratadas como si fueran atributos de individuos y no de grupos.

Lamentablemente, este enfoque individual de los problemas de salud todavía predomina, y ello no solo se traduce en un problema científico-técnico, sino que refleja concepciones más profundas sobre las relaciones entre el individuo y la sociedad. Según estas concepciones, el individuo es la unidad básica de la sociedad y esta se reduce a un mero epifenómeno: la suma de disposiciones, creencias, decisiones y acciones de los individuos que a ella pertenecen (4).

Recientemente, sin embargo, han aumentado en cantidad y calidad los estudios que investigan las relaciones entre la salud de las poblaciones, la desigualdad y el capital social, variables que son esencialmente atributos de sociedades y no de individuos. Estos estudios están demostrando la importancia de tener en cuenta que diferentes niveles de análisis arrojan resultados distintos en relación con los factores que determinan la salud. De hecho, algunos factores que son importantes para explicar diferencias en el estado de salud de los individuos no explican diferencias en la salud de distintos grupos en el seno de una sociedad o entre una sociedad y otra. En otras palabras, no basta sumar los factores determinantes de la salud identificados en estudios de individuos para conocer los factores que determinan la salud a nivel de la sociedad. Por ejemplo, las importantes diferencias de mortalidad constatadas entre clases sociales no pueden ser explicadas por los mismos factores a los que se atribuyen las diferencias entre individuos, ya que si controlamos estos factores (hábito de fumar, dieta, sedentarismo, etc.), las diferencias entre clases permanecen casi inalteradas (5). Confundir los niveles de análisis y tratar de explicar la salud de las poblaciones a partir de los resultados de estudios realizados con individuos equivaldría a aceptar el reverso de la falacia ecológica (6).

La capacidad que hoy tenemos de discernir estos niveles de análisis distintos y de dominar los instrumentos teóricos y metodológicos correspondientes a cada uno de ellos nos permite enfrentar problemas de alta complejidad como la violencia - concebida como problema de salud pública- sin vernos obligados a tratarlos como enfermedades ni limitarnos a identificar los factores de riesgo individuales asociados con ellos. Hablar de la "aplicación" del enfoque de la salud pública (es decir, del enfoque de riesgo) a la violencia no solo es inadecuado porque implica una identificación forzada de la violencia como si fuese una enfermedad, sino también porque supone considerar el enfoque de la salud pública como algo inmutable que se aplica a objetos distintos, que, a la postre, acaban reduciéndose a uno solo. Es difícil resistir la tentación de establecer una analogía con aquel niño a quien se le regala un martillo y que a partir de entonces transforma en clavo todos los objetos.

Si hoy podemos aprehender la complejidad de la violencia como problema de salud pública sin tener que reducirla a la condición de enfermedad es porque ha habido avances importantes en la teoría y práctica de la salud pública. Por otro lado, el entender problemas de salud pública de esta complejidad permite seguir desarrollando el instrumental teórico y metodológico en este campo. El análisis de un problema de esta naturaleza no se lleva a cabo impunemente, pues al realizarlo uno también se transforma.

Mediante este proceso de ampliación de sus temas, disciplinas y enfoques, la salud pública está logrando desarrollarse en la dirección de un nuevo paradigma que incluye, al menos, cuatro elementos clave: la transdisciplinariedad, esto es, la capacidad de construir puentes entre campos disciplinarios distintos; la complejidad, es decir, el desafío que supone lograr un conocimiento efectivo de totalidades complejas sin recurrir a abstracciones simplificadoras; la pluralidad, o sea, una actitud de apertura que rechaza las presuposiciones monolíticas, los monopolios del pensamiento y las aproximaciones controladas, $\mathrm{y}$, finalmente, la praxis o necesidad de que los hallazgos de la ciencia cristalicen en la práctica y de que las explicaciones se den con el propósito de identificar puntos sensibles donde las intervenciones tengan mayor impacto (7).

El estudio multicéntrico que se presenta en este número especial sobre la violencia se inserta en esta concepción de la investigación en salud pública: la que rechaza la reducción de lo complejo a lo simple como única estrategia posible en la ciencia, que intenta unir la simplicidad y la complejidad, superando el dilema entre el pensamiento reductor, que no ve más que los elementos, y el pensamiento globalizador, que no ve más que el todo (8). En este estudio la violencia se aborda desde la perspectiva de la psicología social, a partir de un nivel de análisis individual en el que la violencia se reduce a comportamientos violentos y sus relaciones se analizan mediante atributos individuales como actitudes, ha- 
bilidades y normas culturales interpretadas desde la perspectiva del individuo. Se trata, por lo tanto, de una mirada bastante parcial del fenómeno que no pretende dar cuenta de todas sus dimensiones y que, por el contrario, señala la necesidad de complementarse con otros estudios, desde otras perspectivas y disciplinas que contemplen otros niveles de análisis. De ese modo, mediante aproximaciones sucesivas, se aspira a poder construir modelos explicativos más completos.

A pesar de las limitaciones señaladas, los resultados de los estudios permiten no solo comprender mejor el problema, sino también diseñar algunas intervenciones. Valga recordar que los estudios del nivel individual tienen implicaciones distintas de las que tienen los estudios a nivel poblacional en lo que se refiere al diseño de políticas e intervenciones. Por lo general, las intervenciones que derivan de estudios con individuos se limitan a identificar a los más vulnerables o, como en este caso, a los más propensos a mostrar comportamientos violentos, lo cual permite saber en torno a cuáles es preciso actuar. Sin embargo, no es ese el único tipo de intervención que puede derivarse de los estudios a nivel individual. Aunque los factores individuales son importantes, las intervenciones poblacionales o sociales pueden ser mucho más efectivas y eficientes, ya que es muy difícil reducir la exposición de un grupo específico de individuos a un factor de riesgo determinado sin reducir la exposición de la sociedad como un todo a ese mismo factor. Resulta muy complejo cambiar los comportamientos de individuos en riesgo sin cambiar las normas culturales que los generaron. Incluso si se consigue que algunos de estos individuos cambien, rápidamente serán reemplazados por otros (9).

Sin embargo, la salud pública no puede limitarse a acciones en torno a los factores que determinan la salud a nivel individual, bien sea mediante intervenciones individuales o poblacionales. Los problemas de la salud pública deben abordarse desde la perspectiva de la sociedad. Ello exige diseñar políticas que tomen en cuenta los factores que determinan la salud en este nivel y que reconozcan el carácter esencialmente político y social de los problemas de salud de las colectividades. Por otro lado, se han de articular los diversos sectores de la sociedad para reunir el poder necesario que permita promover cambios radicales que lleguen a la raíz de los problemas. Este enfoque no es nuevo. En realidad, a mediados del siglo XIX destacados salubristas como Virchow pensaban y actuaban de este modo, aunque estuvieran anclados en el paradigma miasmático. Ellos entendían que la "ciencia médica es intrínseca y esencialmente una ciencia social", que las condiciones económicas y sociales ejercen un efecto importante sobre la salud y la enfermedad, y que tales relaciones deben someterse a la investigación científica. Para Virchow, la propia "salud pública" traduce su carácter político y su práctica conduce necesariamente a la intervención en la vida política y social para señalar y eliminar los obstáculos que dificultan el funcionamiento de los procesos vitales y la consecución de la salud de la población (10).

Esta postura fue perdiendo terreno para dar paso a las ideas ilusorias que surgieron posteriormente, según las cuales para lograr la buena salud bastaría con identificar y combatir el agente infeccioso, eliminar el factor de riesgo y, más recientemente, alterar el gen. Por fortuna, una nueva vertiente de la salud pública está cobrando fuerza. Rescatando los avances de la microbiología, la genética y la epidemiología del riesgo, así como los de otras ciencias sociales, dicha vertiente retoma el camino abierto por los salubristas del siglo pasado, camino que hoy en día es posible pavimentar con pruebas científico-técnicas rigurosas y sólidas. Esperamos que este número especial sobre la violencia contribuya modestamente a fortalecer esa nueva visión de la salud pública.

\section{REFERENCIAS}

1. Organización Panamericana de la Salud. La salud en las Américas. Washington, DC: OPS; 1998. (Publicación científica 569).

2. Guerrero R. Resultados de estudio multicéntrico sobre magnitud y costos de la violencia. Presentado en un seminario sobre investigaciones en violencia y salud que se celebró en Harvard University, Boston, MA, el 20 de febrero de 1998.

3. Kawachi I, Kennedy B, Lochner K, Prothrow-Stith D. Social capital, in- come inequality and mortality. Am J Public Health 1997;87:1491-1498.

4. Corin E. The social and cultural matrix of health and disease. En: Evans R, Barer M, Marmor T. Why are some people healthy and others not? New York: Aldine de Gruyter; 1994.

5. Lantz P. Socioeconomic factors, health behaviors and mortality. JAMA 1998; 279:1703-1708.

6. Wilkinson R. Unhealthy societies. New York: Routledge; 1997.

7. Pellegrini A, Almeida-Filho N, Trostle J. La investigación de la salud en
América Latina y el Caribe: tendencias y desafíos. En: Sánchez D, Bazzani R, Gómez S, coord. Prioridades en la investigación de la salud colectiva en América Latina. Montevideo: Trilce; 1998.

8. Morin E. El método. Vol. 2. La vida de la vida. Madrid: Cátedra; 1983.

9. Rose J. The strategy of preventive medicine. Oxford: Oxford University Press; 1992.

10. Rosen G. Da polícia médica à medicina social. Rio de Janeiro: Graal; 1980. 